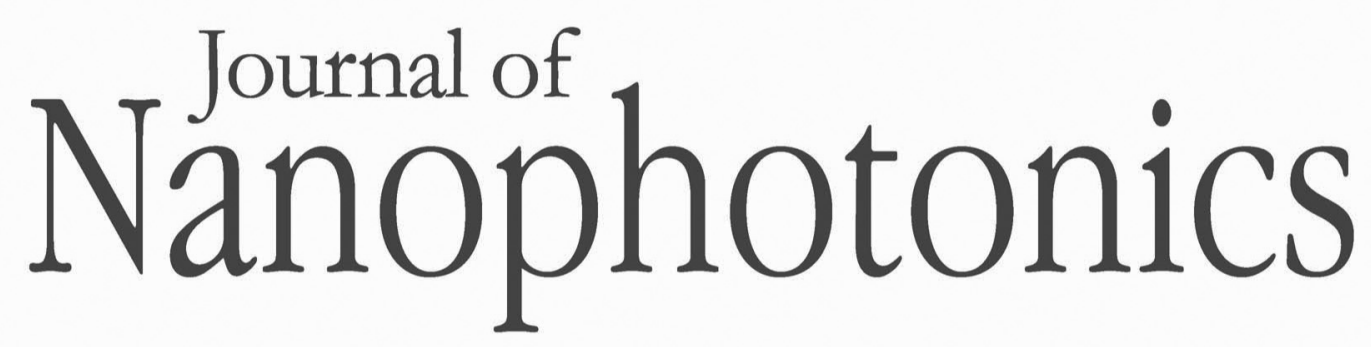

\title{
Simultaneously opening transmission channels with negative and positive phase velocities for the stacked subwavelength apertures in fishnet metamaterials with hybrid unit lattices
}

Atilla Ozgur Cakmak

Evrim Colak

Ekmel Ozbay

\section{O SPIE}




\title{
Simultaneously opening transmission channels with negative and positive phase velocities for the stacked subwavelength apertures in fishnet metamaterials with hybrid unit lattices
}

\author{
Atilla Ozgur Cakmak, Evrim Colak, and Ekmel Ozbay \\ Bilkent University, Department of Physics, Department of Electrical and Electronics \\ Engineering, Nanotechnology Research Center, 06800 Ankara, Turkey \\ atilla@ee.bilkent.edu.tr
}

\begin{abstract}
Hybridization of the unit lattice along the propagation direction was demonstrated to produce a negative-phase-velocity transmission band in the absence of the contributions from the higher diffraction orders for a stacked metallic fishnet grid with subwavelength apertures. This extraordinary transmission band is governed by the stacked resonators. The hybridized unit lattice configurations are not just slight modifications of the configurations with homogenous unit lattices. The volumetric proportions of different dielectric media are a key factor in the partitioned unit lattice for the estimation of the stacking and coupling effects between the resonators. The contribution of the coupling mechanisms enhances the transmission results almost by the same factor for the investigated lattice separations along the propagation direction in hybrid unit cells while the densely stacked resonators yield much higher transmission results, both around the regarding extraordinary transmission band that is associated with the negative phase velocity. A positive-phase-velocity transmission band was also exhibited by the hybridized unit lattice configuration when combined with a cavity resonator. Experimental transmission results of the hybrid configuration supported the theoretical predictions. The hybrid configurations are scalable to the near-infrared regime. () 2012 Society of Photo-Optical Instrumentation Engineers (SPIE). [DOI: 10.1117/1.JNP.6.061608]
\end{abstract}

Keywords: metamaterials; resonators; apertures; diffraction; plasmonics; Fabry-Perot.

Paper 12029SS received Mar. 30, 2012; revised manuscript received Sep. 6, 2012; accepted for publication Sep. 7, 2012; published online Oct. 5, 2012.

\section{Introduction}

The problem of electromagnetic wave transmission through subwavelength apertures had been addressed in 1940s by Bethe. ${ }^{1}$ Bethe had shown that the transmission efficiency scaled as $(r / \lambda)^{4}$ for a subwavelength opening with a radius of $r$ in a perfectly conducting thin screen at the operational wavelength, $\lambda$. Accordingly, the poor transmission figures of the subwavelength apertures presented a challenge for the researchers in the last century. After the pioneering work of Ebbessen et al. $^{2}$ at the turn of the century, the scientific community has started to seek alternative methods to tackle this fundamental problem in physics with the aid of the periodically perforated plates. Several papers have aimed to understand the underlying physics of the extraordinary transmission (EOT) through subwavelength aperture arrays and slits. ${ }^{3-7}$ It was initially thought that the surface plasmons were the main responsible contributors for the EOT at optical wavelengths. In the optical regime, the dielectric and metallic interfaces allowed the propagation of the surface plasmons by exploiting the dispersive characteristics of the metals. The demonstration of the similar phenomenon at microwave frequencies with perfect conductors was explained by making use of the designer surface plasmons. ${ }^{8}$ On the other hand, separate research groups have emphasized the analytical models of the propagating modes through such subwavelength openings. ${ }^{9-11}$ Hence, the EOT is associated with the coupling of the leaky surface waves at both sides of the metallic screen via the evanescent modes of the subwavelength apertures.

0091-3286/2012/\$25.00 @ 2012 SPIE 
Nonetheless, the theory of surface plasmons offers applicable models at optical wavelengths. The analytical dispersion relations can be used to explain the EOT in several examples. As in the case of Ref. 12, the extended surface plasmons are identified to be the cause of the multiple EOT peaks in thin metallic nanoslits when the structure is asymmetric, i.e. the substrate and superstrate around the slits are different dielectrics.

Independently, the quest for obtaining negative refraction has resulted in the realization of the artificial materials called metamaterials that are carefully engineered to yield negative refractive index. Fishnet-like periodic metallic configurations have been favored as a prospective metamaterial building block by the researchers due to its planar manufacturability, reduced transmission losses, and polarization insensitivity. Furthermore, the fishnet metamaterials overcame the magnetic saturation problems, which were heavily suffered by alternative methodologies and this enabled the fishnet based configurations to operate at higher frequencies. ${ }^{13,14}$ In Refs. 15 and 16, it was pointed out that two seemingly distinct phenomena (EOT and negative refraction) manifest themselves in the same type of fishnet designs. Once the connection between the EOT and negative refraction has been formed, the validity of the effective constitutive parameters extracted from the fishnet metamaterials has been discussed in Ref. 17. It was shown in Ref. 18 that the analyses based on the effective parameters are prone to failure for incident angles that are even slightly higher than the normal incidence. Consequently, the homogenized artificial material built out of the stacked fishnet metamaterials are treated in many cases as one dimensional metamaterials with transmission line models similar to the ones proposed in Ref. 19.

Likewise, the electromagnetic wave propagation through stacked fishnet structures is investigated in the present paper. Unlike the previous studies, ${ }^{20,21}$ a hybrid unit lattice along the propagation direction is considered in our structures. Consequently, the unit lattice is divided into sections filled with alternating dielectric media that are sandwiched by fishnet patterned metallic layers. Although it was not intended to study the electromagnetic wave propagation within the framework of the subwavelength apertures, hybridized unit lattices were also exploited in the recent studies and the $L C$ resonator nature of the various similar fishnet configurations were utilized in order to define a metamaterial building block. ${ }^{22-26}$ Another hybrid unit lattice fishnet structure was theoretically studied at optical wavelengths, and the excited surface plasmons from one side were demonstrated to be coupled to the output surface with the aid of the virtual current loops on the metallic structures,${ }^{27}$ where the relation between the transverse $H$-field and induced current loop is similar to the cases studied in Refs. 22 to 26.

In our present work, we show for the first time the clear distinction between the hybridized unit lattice configurations and the homogenous unit lattice designs under certain conditions. We examine the ratios of the volumes occupied by the dielectric materials in the unit lattice with a parametrical study and identify the coupling effects between each metallic fishnet pair. We report and emphasize that the hybridization of the unit lattice is not a minor modification on top of the counterparts with homogenous unit lattices. The negative-phase-velocity (NPV) transmission channel can be managed by the hybridization process. It is also shown that a cavity mode with the usual positive phase velocity is attainable with the same type of stacked fishnet layers. As a result, we show that NPV and positive-phase-velocity (PPV) transmission channels can be opened after certain manipulations at frequency bands in which the former case (homogenous unit lattice) did not allow transmission. The designs are scaled up to operate at near-infrared region. The contribution of the plasmonic effects to the transmission is discussed in order to determine the governing phenomenon that is responsible for the subwavelength wave propagation. The simulations and transfer matrix method based discussions are backed up by experimental validations.

\section{Designs and Simulations}

\subsection{Homogenous Unit Lattice Configurations}

First, we begin by introducing a homogenous unit lattice design shown in Fig. 1(a), which is similar to the topologies considered in Refs. 20 and 21. The relevant dimensions are given in the figure caption. This is the first configuration that we are going to be investigating. The metallic 

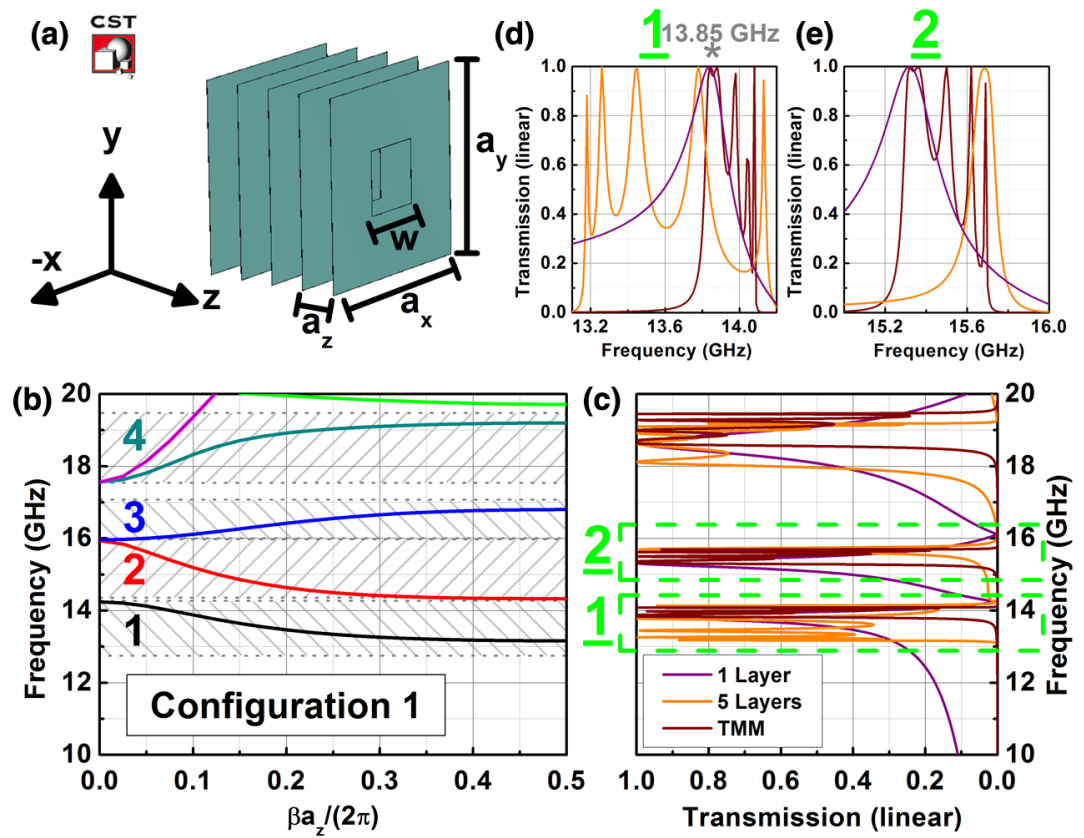

Fig. 1 (a) Stacked metallic layers with subwavelength apertures. $w=7 \mathrm{~mm}, a_{x 1}=a_{y 1}=21 \mathrm{~mm}$, $a_{z}=4 \mathrm{~mm}$. (b) Dispersion diagram of the given configuration, (c) the transmission results for the single layer (purple line) and five-layer structures (orange line). TMM results are also plotted in (c). (d) Zoomed version of the transmission window 1 in (c). (e) Zoomed version of the transmission window 2 in (c). The frequency domain is separated into distinct intervals as shown in the dispersion graph, and these frequency bands are labeled numbers with the corresponding color of the propagating modes.

plate is perforated with aperture arrays in the $x-y$ plane. The metallic layers are separated by air spacings. The distance between each metal layer is fixed to the same value. Thus, such designs fall under the category of homogenous unit lattice configurations. The propagation direction is along the $z$-axis. We carried out our simulations in CST Microwave Studio, which employs finite integration technique (FIT).

The dispersion graph is plotted in Fig. 1(b) in order to distinguish the characteristics of the propagating modes, as in the case of Ref. 28 . Each mode is represented with a different color and the frequency band is partitioned into four regions. The first band approximately resides between $13 \mathrm{GHz}$ and $14 \mathrm{GHz}$ and has a negative slope, $\partial \omega / \partial k<0$, which dictates that the transmission band has negative phase advance characteristics while $\omega$ is the angular frequency. In other words, the phase and group (the direction of the Poynting vector) velocities travel in opposite directions, as it is commonly encountered for metamaterials. The dispersion graph has been calculated for the infinitely extending periodic structure along the propagation direction. Then, the transmission results for the five-layer case in Fig. 1(c) are expected to match the outputs of the dispersion graph.

The cutoff frequency of the individual square shaped holes is $f_{c}=c / 2 w \approx 21.42 \mathrm{GHz}$. This is the lowest propagating mode, $\mathrm{TE}_{10}$ of the square aperture. However, we are confronted with transmission values reaching up to unity in Fig. 1(c), which spans a frequency region from $10 \mathrm{GHz}$ up to $20 \mathrm{GHz}$. Two operational frequency windows from Fig. 1(c) have been zoomed for convenience and replotted in Fig. 1(d) and 1(e). The 1st (2nd) transmission window in Fig. 1(d) [Fig. 1(e)] attends to the 1st (2nd) transmission band that is depicted in the dispersion graph in Fig. 1(b). The same color coding of the curves in Fig. 1(c) have been preserved for its zoomed versions in Fig. 1(d) and 1(e).

The discrepancy between the transmission results of the single (purple line) and five-layer (orange line) configurations is caused by the stacking of the alternating layers as far as the 1st band is concerned in Fig. 1(d). The oscillatory behavior within the 1st transmission band is a classical feature of the widening of the transmission band as soon as the additional layers are 
inserted. Five peaks have emerged inside the 1st transmission band of the five- layer structure due to the periodical arrangement [see Fig. 1(d)]. The effective parameters of the single-layer structure have been retrieved and incorporated into the transfer matrix method (TMM) formalism in order to identify the effects coming from the stacking of the metallic layers. Even though the TMM results fall short in the elucidation of the whole transmission spectrum, the general attributes can be immediately spotted. The TMM results (wine colored line) are illustrated in Fig. 1(c) as well. The ringing inside the 1st transmission band can be more clearly noticed in the zoomed version of the 1st frequency window [see Fig. 1(d)]. We again have five peaks.

We specifically define the appearance of two main effects for the stacked layers. These effects are the stacking and coupling effects that come into the picture for structures with multiple layers. TMM formalism offers a simple method to distinguish the effects coming from the layer stacking and the mutual coupling between the alternating layers. TMM makes use of the single-unit lattice transmission parameters while calculating for the stacked case. Then, the results that are reproducible with the aid of the TMM formalism are recognized as the outcome of the periodic reflections along the propagation direction. These results that are comparable to the (transmission) simulation results for multiple layers exhibit the so-called stacking effect. On the other hand, the results that are not duplicated with the TMM formalism are ascribed to the coupling effects between the stacked layers, i.e., coupling is effective between the layers and a simple stacking effect cannot explain the observed phenomena in the considered case.

TMM results have been recurrently compared with single-layer and five-layer transmission results based on the FIT. Consequently, based on the TMM formalism we can conclude that the narrow EOT band for the single layer (purple line) in Fig. 1(d) is broadened, and the oscillations in the transmission band come into play owing to the stacking of the alternating layers [compare single layer (purple line) and TMM results (wine colored line) in Fig. 1(d)]. Moreover, the flattening of the zero transmission regions with the additional layers is also associated with the stacking effect. Yet, further broadening of the EOT band is a result of the mutual coupling between the stacked resonators [compare five-layer (orange line) and TMM results (wine colored line) again in Fig. 1(d)].

The sudden drop in the transmission levels between the 1st and 2nd transmission bands in Fig. 1(c) is ascribed to the Rayleigh Wood (RW) anomaly. The periodicity dictates that the tangential components of the magnetic and electric fields are set to zero at the walls of the boundaries, which lie on the $y-z$ and $x-z$ planes, respectively. As a result, the unit lattice is in a way placed inside a larger waveguide with perfect magnetic and electric conductor walls, which encapsulates the fishnet configuration and imposes periodicity on the $x-y$ plane. The RW frequency of this relatively larger artificial waveguide is found as $f_{\mathrm{RW} 1}=c / a_{x 1} \approx 14.29 \mathrm{GHz}$, which coincides with the null in the transmission in Fig. 1(c). The EOT that is observed in Refs. 20 and 21 have also been reported just below $f_{\text {RW }}$.

The remaining transmission bands extending beyond $f_{\mathrm{RW}}$ have been out of the main focus in Refs. 20 and 21. Nevertheless, these bands have been portrayed in Fig. 1 in our present study in order to capture the main features of the rest of the transmission bands. The 2 nd band possesses a negative slope as well. Although the detailed examination of these bands [2nd, 3rd, and 4th transmission bands in Fig. 1(b) and 1(c)] are beyond the scope of this work, it is interesting to note that the ringings have not appeared in the FIT-based simulation results (orange line) for the five-layer case in the 2nd band in Fig. 1(e), while the TMM formalism (wine colored line) predicted the recurrence of such peaks within the 2nd frequency window. Further simulations have been performed with 11 stacked layers (results are not shown here). The 2nd transmission band starts to disappear for thicker arrangements in the FIT-based simulation results. The 3rd band is not efficiently coupled to the periodic arrangement and does not contribute effectively to the simulated transmission results, whilst multimode operation is apparent in the 4th band judging from the dispersion results in Fig. 1(b). Overall, only the first two bands have negative phase advance characteristics and the boundaries of the transmission bands agree well with the edges of the bands depicted in the dispersion results [see Fig. 1(b) and 1(c)].

The recipe for achieving EOT band with a negative phase velocity in subwavelength apertures is given in Refs. 20 and 21 with the following relationship between the geometrical parameters, $r<\lambda_{c}<a<\lambda$, where $r$ is the radius and $\lambda_{c}$ is the cutoff wavelength of the subwavelength 
aperture. The lattice constant in the transverse direction is represented with $a$. The first configuration whose dimensions are given in the figure caption of Fig. 1 obeys these geometrical restrictions, and an EOT band appears around $13.84 \mathrm{GHz}$. Conversely, the fishnet metallic layers with the same aperture size and air spacing along the propagation direction do not produce an EOT band as soon as the lattice constants in the transverse direction $\left(a_{x 2}\right.$ and $\left.a_{y 2}\right)$ are reduced to $14 \mathrm{~mm}$, for which the recipe given above is not satisfied any longer.

The regarding dispersion and transmission results are shown in Fig. 2 for the second configuration. The structure exhibits a PPV transmission band between $18 \mathrm{GHz}$ and $20 \mathrm{GHz}$. Actually, this dense configuration acts as a well-known dichroic filter. Several other features presented in the transmission results in Fig. 1 are now lost except for the effect that arises from the stacking of multiple layers. The transmission band undergoes a sharpening with respect to the single layer transmission results with the additional layers and similar oscillatory peaks can be noticed throughout the pass band [compare single (purple line) and five-layer (orange line) transmission results in Fig. 2(b)]. It can be argued that the TMM results (wine colored line) can closely pursue the simulation results up to $19 \mathrm{GHz}$, which is a signature of the reduced mutual coupling between the layers for this configuration. The discrepancies between the TMM results and the FIT-based five-layer transmission results are labeled after $19 \mathrm{GHz}$ in Fig. 2(b). This frequency band marks the region where the retrieved effective parameters of the second configuration can no longer be used to consider the current transmission problem as that of a uniform material.

It is stated in Ref. 11 that the EOT is a consequence of the interactions between the TE/TM modes of the artificial waveguide and the lowest order mode $\left(\mathrm{TE}_{10}\right)$ of the subwavelength aperture as long as $\beta \cdot a_{x, y}>\pi$ for square lattices, where $\beta$ is the propagation constant of the impinging wave at normal incidence. Under such conditions, the fishnet composition brings higher diffraction orders as in the case of a diffraction grating and $\mathrm{TE}_{10}$ is perturbed. Even though this condition is satisfied for both configurations, the RW anomaly is shifted to $f_{\mathrm{RW} 2}=c / a_{x 2} \approx$ $21.42 \mathrm{GHz}$ with the adjustment of the lattice constant in the transverse plane in the second configuration. Therefore, a cross coupling between the modes of the artificial waveguide and that of the subwavelength aperture turns out to be impossible around $14 \mathrm{GHz}$ for the second configuration. The symmetry of the problem allows only even-numbered modes. The lowest modes of the artificial waveguide are $\mathrm{TE}_{20}$ and $\mathrm{TM}_{02}$. These modes are the main contributors of the transmission in the vicinity of the $f_{\mathrm{RW}}$ and their modal admittances for a thin metallic plate can be analytically expressed as $Y_{\mathrm{TE}}=-Y_{0} \sqrt{1-\left(f_{\mathrm{RW}} / f\right)^{2}}$ and $Y_{\mathrm{TM}}=-Y_{0} / \sqrt{1-\left(f_{\mathrm{RW}} / f\right)^{2}}$, where $Y_{0}$ is the admittance of the electromagnetic wave in free space. It can be readily seen that when $f<f_{\mathrm{RW}} Y_{\mathrm{TE}}$ and $Y_{\mathrm{TM}}$ become inductive and capacitive, respectively. Hence, every plate can be considered as a shunt $L C$ tank circuitry with a singularity at $f=f_{\mathrm{RW}}$ and a total impedance $\left(Y_{\mathrm{TE}}+Y_{\mathrm{TM}}\right)^{-1}$. At low frequencies the modal admittance of $\mathrm{TE}_{20}$ becomes dominant and the subwavelength aperture happens to be inductive. As we sweep the frequencies to higher
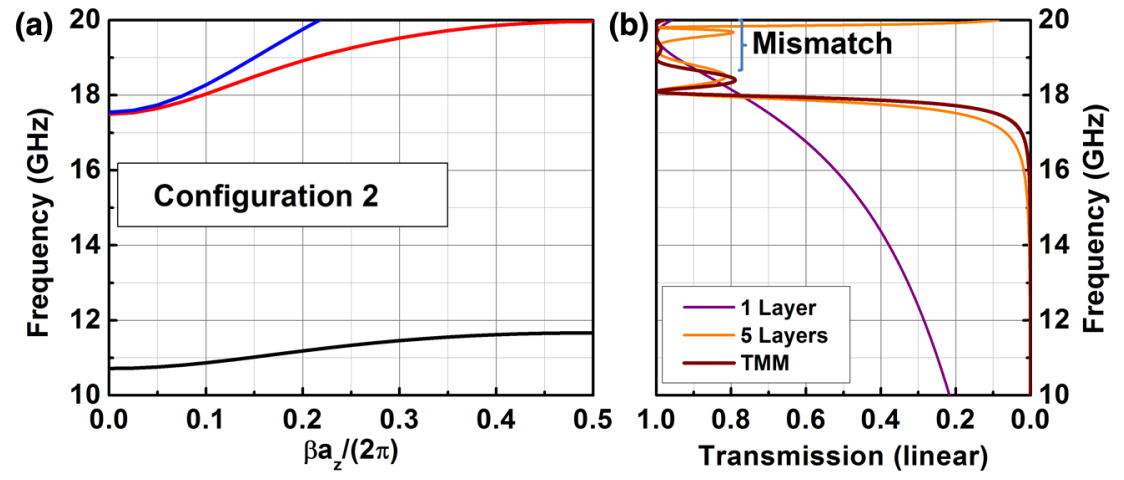

Fig. 2 (a) Dispersion diagram for the configuration with $w=7 \mathrm{~mm}, a_{x 2}=a_{y 2}=14 \mathrm{~mm}$, $a_{z}=4 \mathrm{~mm}$. (b) The transmission results for the single layer (purple line), five-layer structures (orange line) and the TMM results (wine colored line) based on the retrieved parameters for the single layer. The mismatch between the TMM and FIT based results at higher frequencies is labeled. 
values, the susceptance of the regarding modes' modal admittances cancel each other to generate the EOT. For frequency values higher than the EOT band $\mathrm{TM}_{02}$ governs the transmission phenomenon, and the incoming electric field is totally reflected due to the singularity at $f_{\mathrm{RW}}$. This creates a null in the transmission, which we have considered within the framework of the RW anomaly.

The electric field components of the $\mathrm{TM}_{02}$ mode has the following form

$$
\begin{aligned}
& E_{x} \propto \sin \left(m \pi x / a_{x}\right) \sin \left(n \pi y / a_{y}\right) \quad E_{y} \propto \cos \left(m \pi x / a_{x}\right) \cos \left(n \pi y / a_{y}\right) \\
& E_{z} \propto \cos \left(m \pi x / a_{x}\right) \sin \left(n \pi y / a_{y}\right),
\end{aligned}
$$

where the mode numbers are represented with $m$ and $n$. The steady state field components are compared in Fig. 3 for both cases that are considered in Figs. 1 and 2. On the left-hand side, we have the field components of the dense configuration (second configuration) ( $a_{x 2}=$ $a_{y 2}=14 \mathrm{~mm}$ ) at $18.15 \mathrm{GHz}$, whereas Fig. 3(d)-3(f) depicts the conditions for the original case (first configuration) in Fig. $1\left(a_{x 1}=a_{y 1}=21 \mathrm{~mm}\right)$ at $13.85 \mathrm{GHz}$. These frequency values are selected, because they reside inside the transmission bands of the considered configurations. As Eq. (1) implies, we do not have either $x$ component of the electric field or $y$ component of the magnetic field around the EOT band. Furthermore, $E_{z}$ does not possess a variation along $x$-axis but has an odd symmetry around $x$-axis in accordance with Eq. (1), as it is shown in the small inset picture in Fig. 3(e), where the fields are plotted on $y-z$ plane.

The red thick arrows in Fig. 3(a) and 3(d) indicate the direction of the phase velocity inside the structures while the launched field is propagating in $+z$ direction. The negative phase advance in Fig. 3(d) can be monitored in the linked movie file (Video 2) and can be compared with the case depicted in Fig. 3(a) (Video 1), for which the ordinary positive phase advancing propagation is taking place. The most significant difference in the field distributions can be visualized in the $z$ and $x$ components of the electric and magnetic fields, respectively. The fields are in the propagation regime of the dichroic filter's pass band and travel with less harmony in Fig. 3(b) in comparison to the case given in Fig. 3(e). The fields are intensified at the edges of the stacked apertures owing to the diffractions at these discontinuities on the surface of the perfect

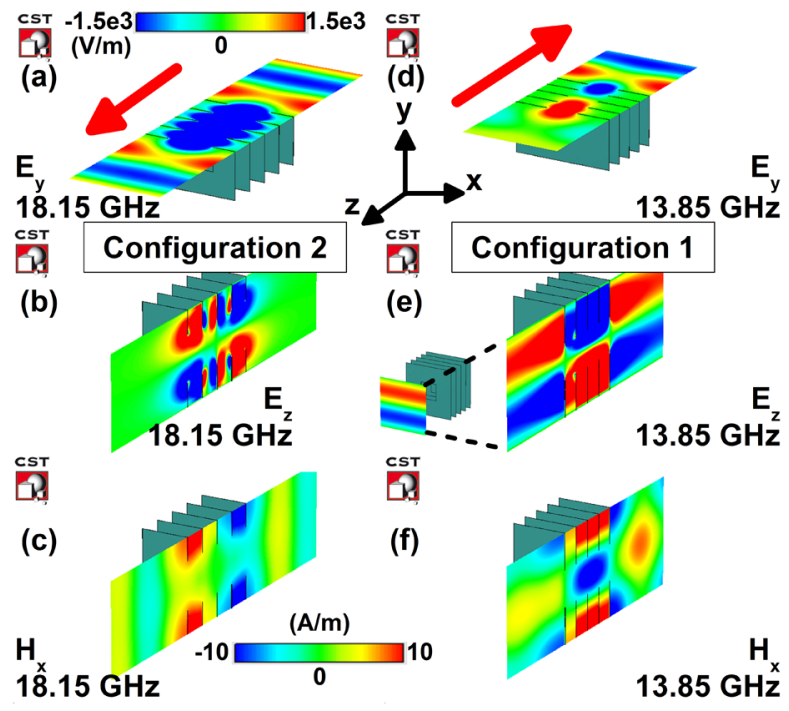

Fig. 3 The steady state field distributions at $18.15 \mathrm{GHz}$ for the dense configuration $\left(a_{x 2}=a_{y 2}=14 \mathrm{~mm}\right)$ are given on the left-hand side, whereas the field distributions at $13.85 \mathrm{GHz}$ are shown on right-hand side for the first configuration $\left(a_{x 1}=a_{y 1}=21 \mathrm{~mm}\right)$. CST Microwave Studio offers the field maps in the form of $\left|E_{y, z}\right| \times e^{-j \varphi}$ and $\left|H_{x}\right| \times e^{-j \varphi}$ for electric and magnetic fields, respectively. The given plots are the captured scenes at an instantaneous phase, $\phi . E_{z}$ on the $x-z$ cut plane in (a) and (d) (Videos 1 and 2), $E_{y}$ on the $y-z$ cut plane in (b) and (e) and $H_{x}$ on the $y-z$ cut plane in (c) and (f). The arrows in (a) and (d) indicate the directions of the phase velocities. (Video 1, MPEG, 825 KB) [URL: http://dx.doi.org/10.1117/1.JNP.6.1.061608.1] (Video 2, MPEG, 826 KB) [URL: http://dx.doi.org/10.1117/1.JNP.6.1.061608.2]. 
conductor screens in Fig. 3(b), and naturally the fields are not scattered uniformly, which results in comparably abrupt field distributions between the plates along the propagation direction. On the other hand, the phase information of the electric field is preserved in Fig. 3(e), and the fields are rather uniformly distributed between the plates, which enables one to define transmission line parameters along the propagation direction. The same situation is also true for the magnetic fields in Fig. 3(f). In contrast to the case in Fig. 3(c), the magnetic fields are uniformly shared. Thus, the air spacing between the metallic plates plays the role of the series distributed capacitors while the subwavelength holes are predominantly inductive in the vicinity of the EOT band, as it is discussed. Then, as a combination of the shunt inductors and series distributed capacitors the backward-wave transmission line models are applicable to understand the wave propagation in Fig. 3(d)-3(f). ${ }^{15}$

\subsection{Opening a PPV Defect-Based Transmission Channel}

It is possible to create a cavity mode for the dense configuration ( $a_{x 2}=a_{y 2}=14 \mathrm{~mm}$ ) following the formalism in Ref. 29. Once a single layer is taken away from the middle of the five-layer system, a Fabry-Perot (FP)-based cavity is formed. The field distribution and the new transmission spectrum are shown in Fig. 4(a) and 4(b), respectively. The defect peak appears at $15.4 \mathrm{GHz}$ with a $Q$ factor of 128 [inspect the peak value of blue line in Fig. 4(b)]. This frequency band originally did not allow transmission, as it was depicted in Fig. 2(b). The symmetric field distributions and the intensified fields inside the cavity are evident in Fig. 4(a), which are the signatures of the FP based cavity modes. Moreover, the reflectivity of the two-layer structures can be estimated to be $r \times e^{-j \psi}$ for every frequency. As soon as these two-layer structures are assumed to be the mirrors of the FP cavity, the following condition has to be satisfied to sustain the constructive interference mechanism inside the cavity structure

$$
\Phi=\beta \cdot L+\psi=m \cdot \pi
$$

where $L$ is the cavity length, it is equal to $a_{z}$ in our case and $m$ is an integer. The extracted reflectivity values for two-layer mirrors have been used in order to find out the frequency at which Eq. (2) is satisfied. The results are plotted in green in Fig. 4(b). The integer solution

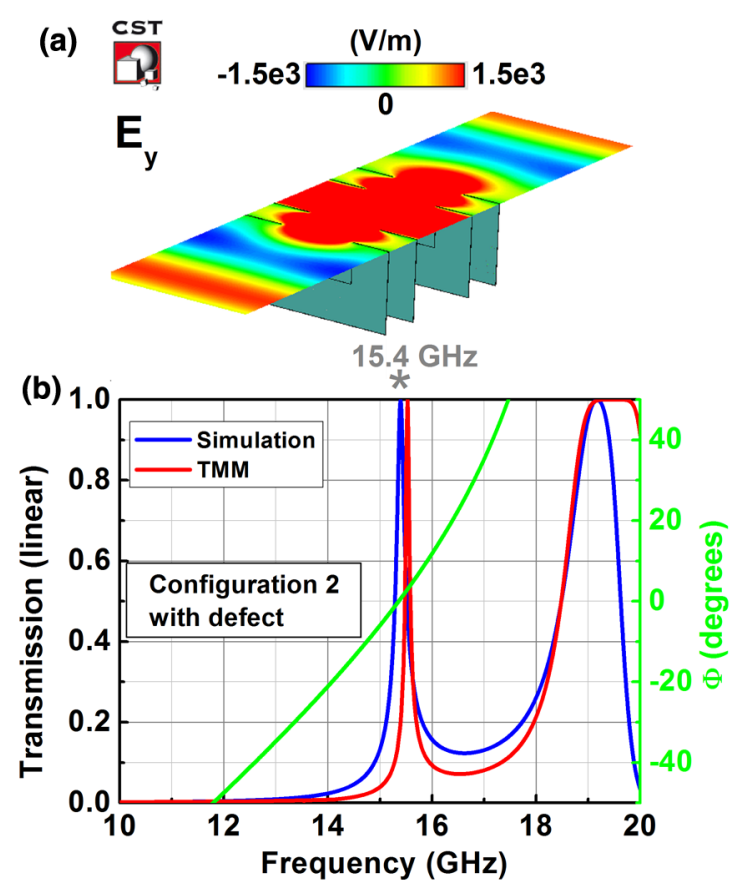

Fig. 4 (a) The steady state electric field distribution at $15.4 \mathrm{GHz}$, (b) transmission results for fivelayer structure (blue line) and TMM results (red line) together with the phase value $(\Phi)$ calculated from Eq. (2) for every frequency value (green line). 
of the $m$ parameter $(m=0)$ coincides with the defect transmission peak that is illustrated on the same graph, which removes the doubts about the origins of the opened PPV transmission channel.

Additionally, the TMM formalism is exploited in order to determine the defect peak in the transmission spectrum (red line). The same retrieved parameters that are used to reproduce the transmission spectrum in Fig. 2(b) have been utilized. Subsequently, the defect peak is detected at $15.54 \mathrm{GHz}$, which points to a $0.14 \mathrm{GHz}$ shift with respect to the simulation results for the five-layer case. Figure 4(b) once again shows that the TMM formalism can be adapted till $19 \mathrm{GHz}$ in correlation with the outputs of Fig. 2(b). TMM results suggest an enhanced $Q$ factor for the cavity mode. The broadening in the defect transmission peak is associated with the mutual coupling between the alternating metallic layers.

\subsection{Opening an NPV Transmission Channel in Hybridized Fishnet Unit Lattices}

In this section, we will discuss how to achieve an NPV transmission channel from the dense fishnet configuration (second configuration), and we will emphasize the differences in the underlying physics with regard to the EOT accompanied by the evanescent TE/TM modes of the artificial waveguide, which was considered in Sec. 2.1. We can break the homogeneity of the unit lattice spacing along the propagation direction by inserting a second dielectric (other than air) at the back of the fishnet layers. Teflon is selected as the substrate due to its low losses in the microwave regime. The permittivity of Teflon substrate is $\varepsilon=2.16$, and it has a loss tangent of $\tan (\delta)=0.005$ and a thickness of $t=1 \mathrm{~mm}$.

The transmission spectrum of the dielectric loaded second configuration is shown in Fig. 5(b) for five layers. As it can also be inspected from the regarding dispersion curves in Fig. 5(a), the pass band of the dichroic filter is shifted toward lower frequencies with the addition of the dielectric medium [compare Figs. 2(b) with 5(b)]. This is nothing but a red shift that occurs by the addition of the higher permittivity into the unit lattice. The bandwidth of the pass band is intact,
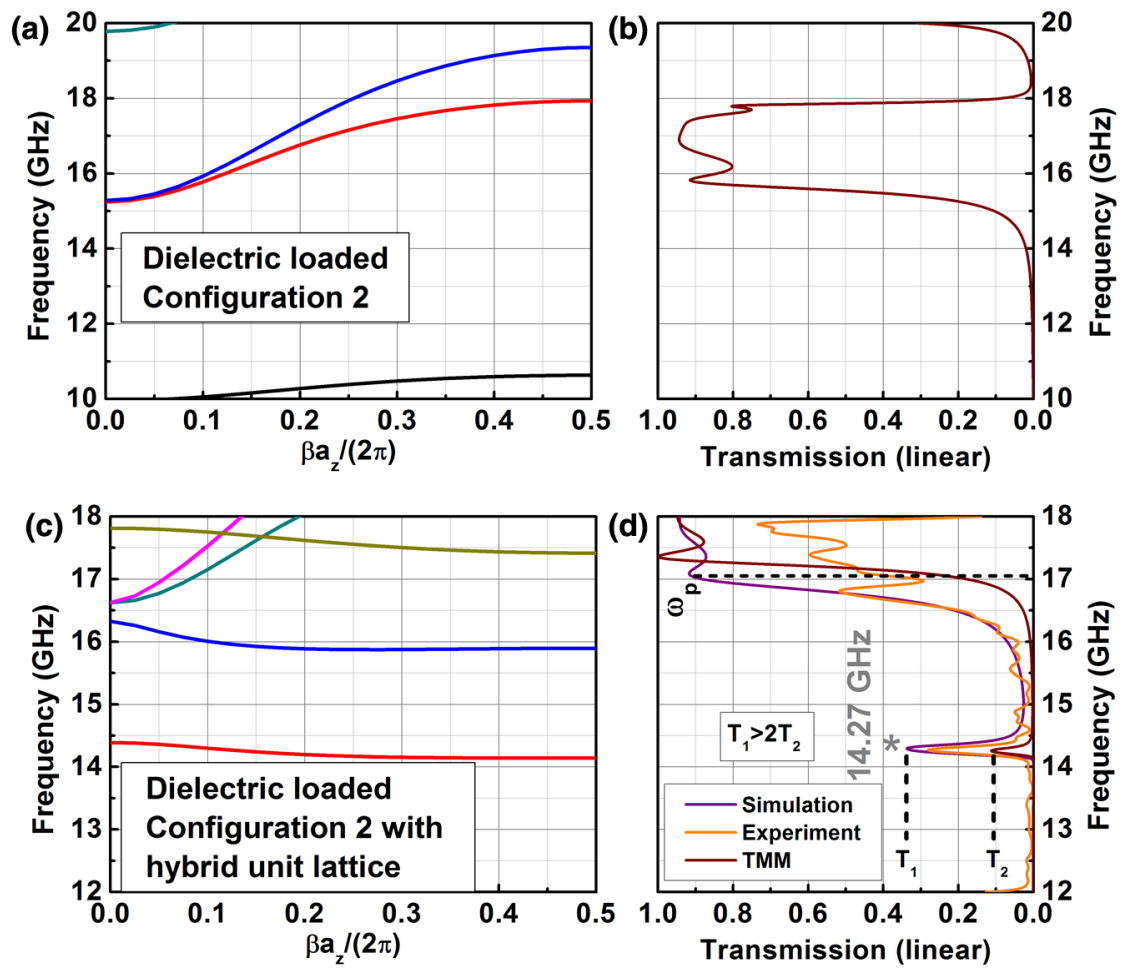

Fig. 5 (a) Dispersion diagram for the dense configuration with dielectric loading and (b) the calculated transmission spectrum for the same sample. (c) Dispersion diagram when a second metallic grid is stacked at the back of the dielectric medium and (d) the calculated (purple line), measured (orange line) and TMM formalism based (wine colored line) transmission results. 
and it is again around $2 \mathrm{GHz}$. The impedance matching at the fishnet surface has been altered, and the transmission values have decreased to $90 \%$ rather than the unity transmission as in the case of the former situation without the dielectric loading.

The NPV transmission band is opened as soon as we stack a second fishnet metallic layer at the back side of the dielectric medium, as it can be seen in Fig. 5(d). Thus, the NPV transmission band is observed around $14.27 \mathrm{GHz}$, again for five layers. The negative slope of the corresponding band (red-colored band) in Fig. 5(c) reveals that the transmission peak observed at this particular frequency is an NPV transmission channel. The PPV pass band of the dichroic filter is reshifted back to higher frequencies. Actually, the starting edge of the PPV pass band can be regarded as the plasma frequency $\left(\omega_{p}\right)$ of the metallic grid. ${ }^{8}$ The effective permittivity of this metallic grid is below zero and thereby does not allow transmission before the plasma frequency. The addition of the second metallic grid at the back of the dielectric increased the density of metals in the unit volume and resulted in the increase of the plasma frequency of the system in Fig. 5(d) in comparison to Fig. 5(b). The experiments have also been carried out for the same configuration with conventional 12.4 to $18 \mathrm{GHz}$ horn antennas that are connected to HP 8720B network analyzer. The experimental results (orange line) are in good agreement with the numerical expectations (purple line). TMM results based on the single layer configuration predict a relatively lower transmission at the NPV band and a higher plasma frequency value. This point will be revisited in the following sections.

The field maps and surface currents at $14.27 \mathrm{GHz}$ (left-hand side) and $18.15 \mathrm{GHz}$ (righthand side) are presented in Fig. 6 for the hybrid configuration. These are two frequencies selected from the relevant NPV and PPV transmission bands, respectively. The attached movie files (Video 3 and 4) once again depict the reverse and forward phase propagation inside the stacked units in Fig. 6(a) and 6(e), correspondingly. The field components at $18.15 \mathrm{GHz}$ bear resemblance to the field distributions that are already considered for the previous cases in Fig. 3(a)-3(c). Nevertheless, the surface currents and field components for the NPV transmission band have striking differences. The antiparallel surface currents on each side of the

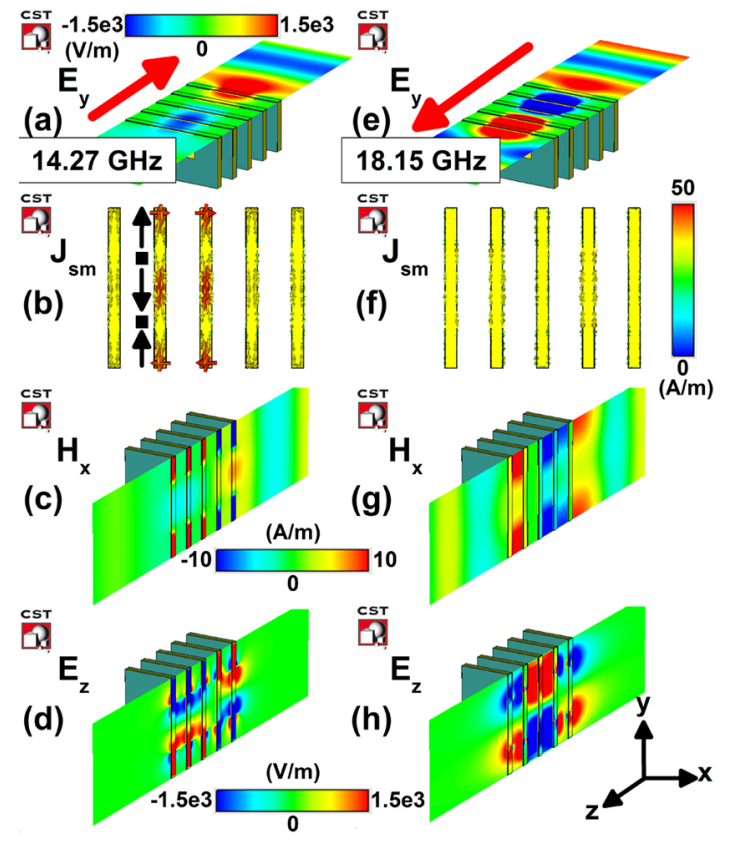

Fig. 6 The steady state field and surface current distributions for the dense configuration. The electric field component $\left(E_{y}\right)$ is plotted at (a) $14.27 \mathrm{GHz}$ (Video 3) and (e) $18.15 \mathrm{GHz}$ (Video 4). The arrows in (a) and (e) indicate the directions of the phase velocities. The surface currents calculated at (b) $14.27 \mathrm{GHz}$ and (f) $18.15 \mathrm{GHz}$. The directions of the surface currents in (b) are highlighted with additional arrows for the designated side. The magnetic field component $\left(H_{x}\right)$ is plotted at (c) $14.27 \mathrm{GHz}$ and (g) $18.15 \mathrm{GHz}$. The electric field component $\left(E_{z}\right)$ is plotted at (c) $14.27 \mathrm{GHz}$ and (g) $18.15 \mathrm{GHz}$. (Video 3, MPEG, 829 KB) [URL: http://dx.doi.org/10.1117/1.JNP.6.1.061608 .3] (Video 4, MPEG, 835 KB) [URL: http://dx.doi.org/10.1117/1.JNP.6.1.061608.4]. 
metallic fishnet patterns are shown in Fig. 6(b) from a side view on the $y-z$ plane. These circulating currents enable us to define a virtual current loop. On the other hand, the surface currents turn out to be parallel to each other for the PPV transmission band in Fig. 6(f). The existence of such a virtual current loop amplifies the circulated magnetic fields $\left(H_{x}\right)$ inside the dielectric medium, as shown in Fig. 6(c). The electric field component $\left(E_{z}\right)$ in Fig. 6(d) is also mainly confined to the dielectric medium, whereas the propagating field components are primarily localized in the air spacings rather than the substrate for the PPV transmission band. Consequently, the hybridized unit lattice boils down to a series of stacked coupled resonators within the NPV transmission band.

Then, we can define quasi-static inductance and capacitance formulae for these resonators. The formalism is presented in Ref. 26 . The capacitance value can be easily expressed by taking into account the parallel plate capacitance formation between the faces of the metallic patterns $C \propto \varepsilon a_{x} a_{y} / t$. The detailed calculation of the total inductance is also given in Ref. 26. A careful examination of the surface currents in Fig. 6(b) shows that there are two inductors parallel to each other. These inductors that are modeled for the walls of the aperture carry the surface currents in two different directions, as designated by the arrows in Fig. 6(b), and the surface currents meet at the square shaped nodes. Then, the total inductance becomes $\left(L_{1} \| L_{2}\right)^{-1}$, where $L_{1} \propto\left(a_{y 2}-w\right) t / a_{x 2}$ and $L_{2} \propto \mathrm{wt} /\left(a_{x 2}-w\right)$. Finally, the overall resonance frequency can be found as:

$$
f_{R}=\frac{1}{2 \pi \sqrt{\mathrm{LC}}} \propto \sqrt{\frac{1}{\varepsilon} \times\left[\frac{1}{a_{y 2}\left(a_{y 2}-w\right)}+\frac{\left(a_{x 2}-w\right)}{a_{x 2} a_{y 2} w}\right]} .
$$

The reported resonance frequencies are $21 \mathrm{GHz}$ and $13 \mathrm{GHz}$ in Refs. 22 and 26 for the fishnet designs with the relevant dimensions, respectively. By making use of the proportionality in Eq. (3), our envisaged resonance frequency turns out to be $14.22 \mathrm{GHz}$, which is a very close value to the mentioned peak location of the NPV band.

These quasi-static definitions for the modeled inductance and capacitance of the subwavelength aperture can only hold for the resonator type structures. The proportionality does not hold for the first configuration ( $w=7 \mathrm{~mm}, a_{x 1}=a_{y 1}=21 \mathrm{~mm}$ ) even if its unit lattice is hybridized along the propagation direction, as we will be discussing in the proceeding parts. Another drawback of the simple resonator formulation in Eq. (3) is the insensitivity of the resonance frequency to the thickness of the inserted dielectric slab. We cannot indefinitely increase the substrate thickness. The ratio of the volumes occupied in the unit lattice for the two dielectric layers plays a key role in the coupling mechanisms for the transmission through the stacked layers. In summary, Eq. (3) offers a first-order approximation of the resonance frequency. Yet, the restrictions on the surface currents and field maps have to be considered carefully before blindly adapting the resonance formalism. Accordingly, we are obliged to take into account the higher order evanescent modes and their dynamic modal expressions (as a function of frequency) while analyzing the EOT band in the homogenous lattice configuration.

\subsection{Differences Between the NPV Transmission Regimes}

So far, two transmission bands with the negative phase advance characteristics have appeared in the dispersion graphs of Figs. 1(b) and 5(c). In this part, we will be focusing on the aspects that govern the NPV transmission band for the considered configurations with different lattice constants on the transversal plane. First of all, it can be noticed that the stacking effect did not yield ringing inside the NPV transmission band for the hybrid configuration in Fig. 5(d). The reason is the confinement of the propagating fields inside the dielectric substrate. The dense configuration is a series of stacked resonators and the transmission phenomenon is dominated by the coupling between these resonator structures. Hence, the peak value of the NPV transmission band computed by the FIT $\left(T_{1}\right)$ is elevated by more than two times in comparison to the TMM results $\left(T_{2}\right)$ owing to this particular coupling mechanism, as it is shown again in Fig. 5(d). On the other hand, the transmission figures already reach unity or close to perfect transmission with the TMM formalism, as it can be seen in the first zoomed window in Fig. 1(d). 

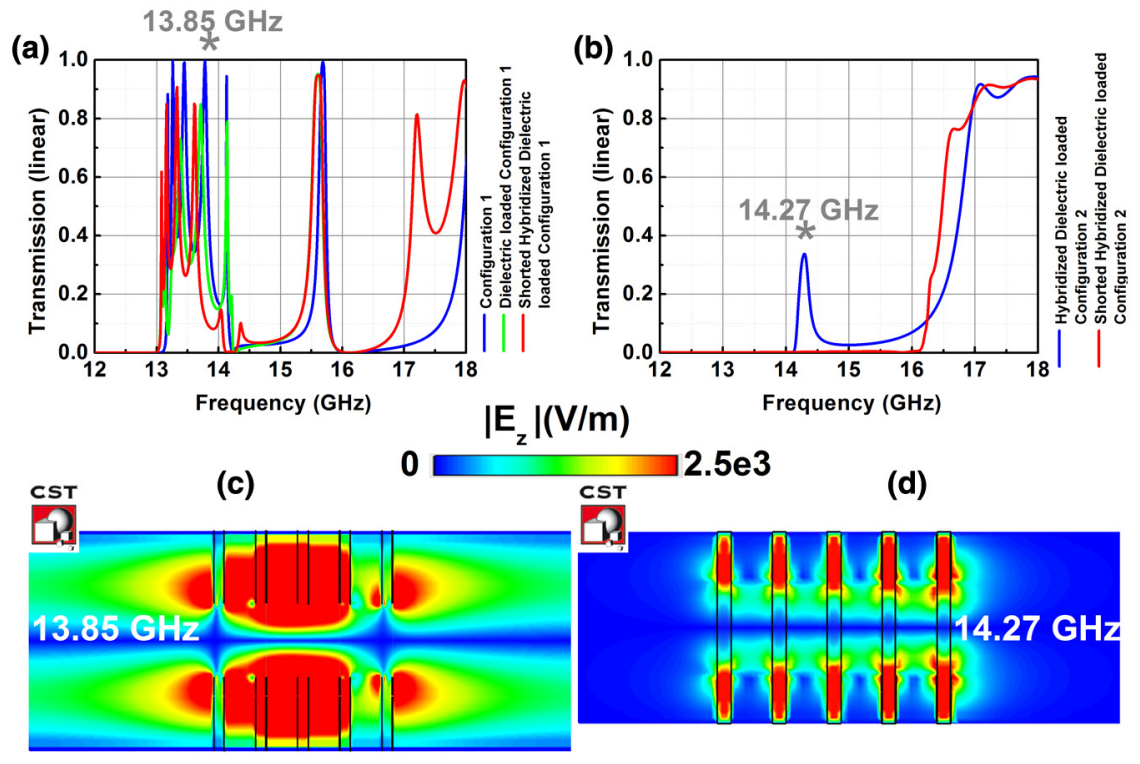

Fig. 7 Transmission spectrum for (a) the first configuration (blue line), the dielectric substrate loaded first configuration (green line) and the shorted hybridized original configuration (red line). Transmission spectrum for the (b) dense configuration (blue line) and its shorted version (red line). The field maps for the average electric field component $\left(E_{z}\right)$ for (c) the original case at 13.85 GHz and (d) the dense configuration at $14.27 \mathrm{GHz}$. The average field values extracted from CST Microwave Studio are different from the previously given steady state field maps. Unlike the previous cases, which were plotted at a random phase value, the fields undergo an averaging within the time period of the incoming wave.

Figure 7(a) depicts the scenario when the first configuration's unit lattice is hybridized along the propagation direction by following the same steps applied to the dense case, which is studied in Fig. 5. In fact, Refs. 30-32 dealt with the situations when the subwavelength aperture is loaded with a dielectric medium. The dielectric loading was realized in order to match the impedance at the surfaces of the fishnet samples. The dielectric slab suppressed the RW anomaly and dramatically changed the transmission spectrum by reducing the maximum attainable transmission value in rectangular lattices (rectangular lattice is achieved in the transversal plane by selecting $a_{x} \neq a_{y}$ ) depending on the thickness and permittivity of the substrate. On the contrary, in our case the transmission spectrum is not fundamentally modified with the addition of the dielectric substrate, except for the slight shifts [compare blue and green lines in Fig. 7(a)].

We used a finite array in our experiments and carried out the measurements in the near field zone. Hence, we were still able to witness the NPV transmission band for the dense configuration regardless of the distance between the transmitter and receiver antennas. In contrast, the performance of the measurements were severely degraded for finite arrays, and the researchers were compelled to work in the Fraunhofer zone since the evanescent modes interacting with the subwavelength aperture array had long propagation lengths for the first configuration. ${ }^{32}$ The resonator nature of the dense configuration removed the necessity for long measurement distances in the experiments.

The most critical distinction appears when we short both of the hybrid fishnet configurations, i.e., the hybrid forms of the configurations 1 and 2. The metal patterns on both surfaces are shorted with small metallic inclusions at the corners of the layers. The dielectric medium between the metallic layers is still present. Then, the virtual current loop is also broken with the shortening of the fishnet structures on both surfaces of the metallic layers. The NPV transmission band automatically disappears, as it is shown in Fig. 7(b) [compare red and blue colored lines], whereas the shortening has little effect on the transmission spectrum for the first configuration which is depicted in Fig. 1(a), being hybridized. The NPV EOT band persistently stays in Fig. 7(a) (red line) for the first configuration, since the EOT phenomenon is governed by the modal expressions of the higher diffraction orders. The average electric field components $\left(E_{z}\right)$ 
are illustrated in Fig. 7(c) and 7(d) at $13.85 \mathrm{GHz}$ and $14.27 \mathrm{GHz}$, respectively. The specific location of these frequencies are indicated in Fig. 7(a) and 7(b). It should be once again stressed that $E_{z}$ is rather uniformly shared between the metallic plates, which results in a transmission line with distributed capacitances for the first configuration. Alternatively, $E_{z}$ is localized to the substrate regions because of the resonance nature of the stacked formations in the dense case in Fig. 7(d).

\subsection{Effect of Coupling Between Alternating Layers for the Dense Configuration}

The interaction between the electric fields of the localized resonators is evident in Fig. 7(d). The field maps for three different lattice spacing values have also been illustrated in Fig. 8 for the hybrid dielectric loaded second configuration. The corresponding transmission spectra and dispersion plots in Fig. 9 have to be evaluated along with the field maps. As it was pointed out earlier, the coupling between the stacked resonators causes an increase in the transmission figures of the NPV band. This is clearly seen in Fig. 9(b) when we compare the transmission levels for $a_{z}=2 \mathrm{~mm}$ (red line) and $a_{z}=6 \mathrm{~mm}$ (blue line). The former situation transmits slightly more than three times at the NPV band $\left(T_{3}>3 T_{4}\right)$. Approximately, the same amount of ratios between the peak levels of the considered two cases can be spotted in Fig. 9(a) as a result of the TMM formalism $\left(T_{5}>3 T_{6}\right)$. However, there is also another two times difference between the TMM and five-layer simulations, which is a direct outcome of the coupling effect $\left(T_{3}>2 T_{5}\right.$, $T_{4}>2 T_{6}$ ). Under these conditions, the retrieved effective parameters for the single layer may not be suitable for the representation of the stacked case (owing to the coupling) and a convergence in the effective parameters cannot be met rapidly even at normal incidence, as Ref. 33 also suggests.

The NPV transmission band peak resides at $14.21 \mathrm{GHz}, 14.27 \mathrm{GHz}$, and $14.38 \mathrm{GHz}$ for $a_{z}=6 \mathrm{~mm}, a_{z}=4 \mathrm{~mm}$ and $a_{z}=2 \mathrm{~mm}$ cases, respectively. TMM results are very close to these values. The NPV transmission band's peak experiences a blue shift due to the compact stacking of the alternating layers. The NPV transmission band's peak is located at $14.19 \mathrm{GHz}$ for the single-layer transmission results (not shown here). The relatively isolated case, $a_{z}=6 \mathrm{~mm}$, produces the closest results to the single-layer transmission. The blue shift at the peak of the NPV transmission band can also be ascribed to the decrease in the average magnetic flux in each layer when the interspacing distance gets smaller. Figure 8 portrays the magnetic field values inside the resonator structures for different $a_{z}$. The magnetic fields are equally shared between the resonators when $a_{z}=2 \mathrm{~mm}$ in Fig. 8(d) whereas the intensified magnetic fields are encapsulated by the first layers in Fig. 8(b). Hence, the average magnetic field value is higher for $a_{z}=$ $6 \mathrm{~mm}$ and thereby the inductance of the resonator system is large in comparison to $a_{z}=2 \mathrm{~mm}$. The larger inductances yield NPV transmission bands at lower frequencies.

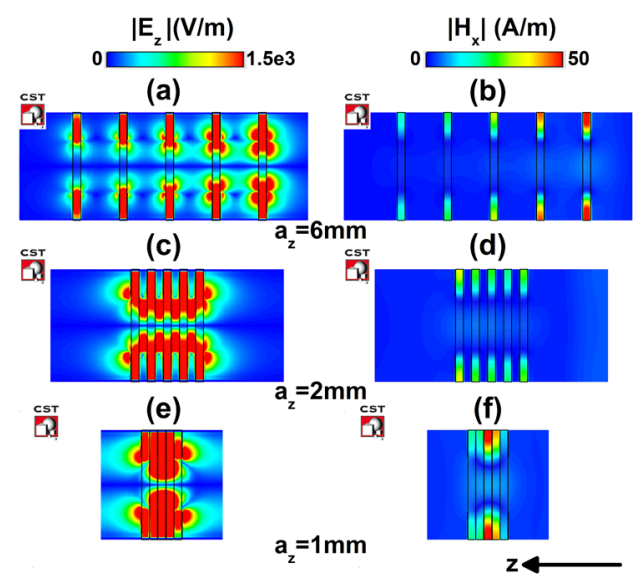

Fig. 8 The field maps for the average electric field component $\left(E_{z}\right)$ and the magnetic field component $\left(H_{x}\right)$ are given on the left-hand side and right-hand side of the figures, respectively. (a) $E_{z}$ and (b) $H_{x}$ at $14.27 \mathrm{GHz}$ for $a_{z}=6 \mathrm{~mm}$. (c) $E_{z}$ and (d) $H_{x}$ at $14.38 \mathrm{GHz}$ for $a_{z}=2 \mathrm{~mm}$. (e) $E_{z}$ and (f) $H_{x}$ at $13.68 \mathrm{GHz}$ for $a_{z}=1 \mathrm{~mm}$. 

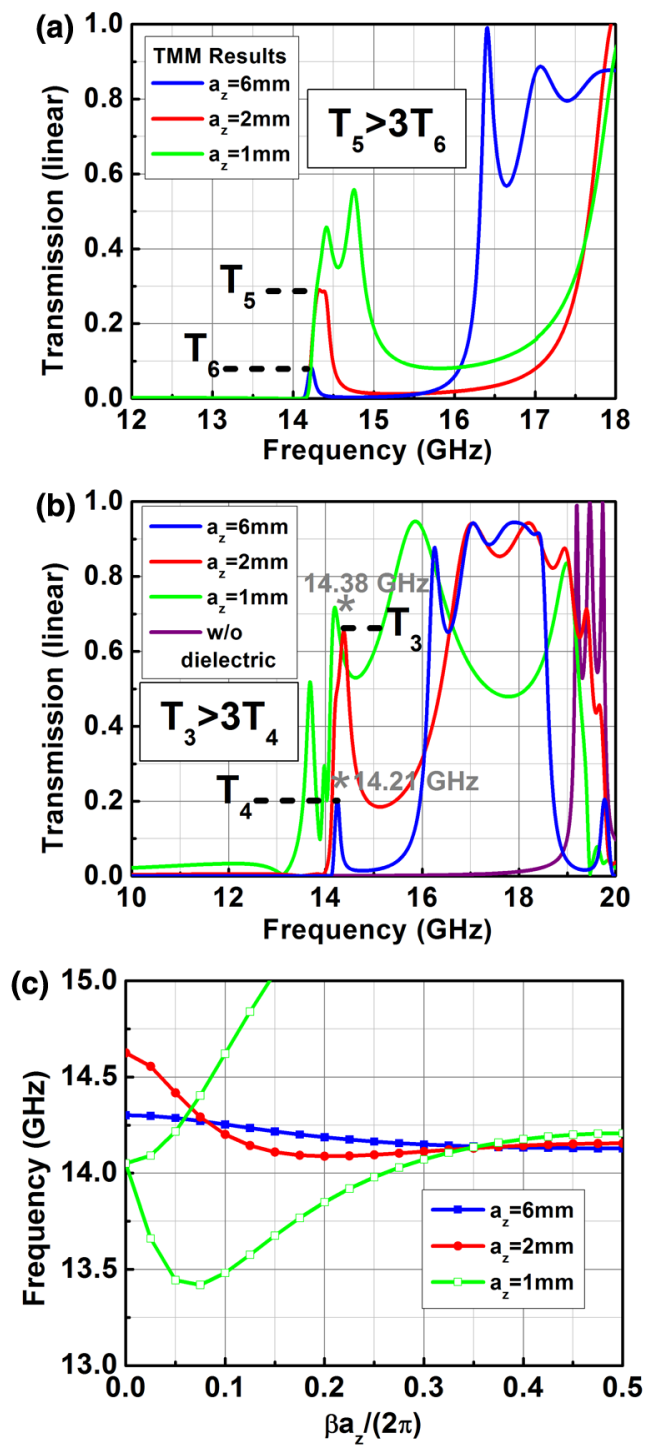

Fig. 9 (a) Transmission spectrum based on TMM results for $a_{z}=6 \mathrm{~mm}$ (blue line), $a_{z}=2 \mathrm{~mm}$ (red line) and $a_{z}=1 \mathrm{~mm}$ (green line). (b) Calculated transmission spectrum for $a_{z}=6 \mathrm{~mm}$ (blue line), $a_{z}=2 \mathrm{~mm}$ (red line), $a_{z}=1 \mathrm{~mm}$ (green line) and the case without dielectric loading (purple line). (c) Dispersion diagram for $a_{z}=6 \mathrm{~mm}$ (blue line), $a_{z}=2 \mathrm{~mm}$ (red line) and $a_{z}=1 \mathrm{~mm}$ (green line).

The bandwidth of the NPV transmission band is also increased for decreasing interseparation distance, as it can be noticed both in Fig. 9(a) and 9(b). In other words, the NPV band gets broader when we decrease the fraction of the volume occupied by air spacings. TMM results also confirm this broadening effect. Accordingly, we can conclude that the broadening is a consequence of the smaller inter-separation distance between the alternating layers, i.e., the stacking effect, and it is not a direct consequence of the coupling between the fishnet resonators.

The plasma frequency is lowered down with larger separation distances between unit lattices in Fig. 9(b). This is consistent with our earlier claims concerning the change in the metal density per unit volume. Nevertheless, the retrieved effective parameters are liable to errors while describing the transmission spectrum for the PPV band, as it can be noticed when the PPV transmission bands in Fig. 9(a) and 9(b) are compared. Therefore, the TMM results can be mainly utilized to understand the transmission effects around the NPV band. Lastly, the Teflon substrate between the facing fishnet patterns is replaced with air as the dielectric loading and this new configuration with air is also simulated and the results are plotted (purple line) in Fig. 9(b). 
The unit lattice is still partitioned into two sections and this configuration can still be considered to possess a hybrid form. However, the NPV transmission band cannot be spotted. The dielectric medium that lies inside the resonator plays a key role in tuning the resonance frequency, as Eq. (3) implies. For air, the NPV transmission band cannot be adjusted to lower frequencies and only the PPV band is witnessed in the transmission spectrum.

As the last scenario, the case without the air spacing is investigated. Then, the unit lattice separation becomes $a_{z}=1 \mathrm{~mm}\left(a_{z}=t+2 t_{\text {metal }}\right.$ and $t=1 \mathrm{~mm}, t_{\text {metal }}=20 \mu \mathrm{m}$. $a_{z}$ is rounded to $1 \mathrm{~mm}$ ). For such an arrangement, the unit lattice is again homogenous. The transmission results (green line) are given in Fig. 9(b). The formerly pronounced NPV band is degenerated. Now, the plasma frequency is shifted down and the PPV transmission band is merged with the NPV transmission band. A multimode behavior accompanies this configuration unlike the rest of the considered hybrid cases. The dispersion graph in Fig. 9(c) predicts both negative and positive phase advancing modes at the same frequency, since the regarding bands are merged. TMM results can predict the degeneration of the modes for $a_{z}=1 \mathrm{~mm}$ case up to some extent (notice the double humps inside the NPV transmission band and the elevated transmission figures between the NPV and PPV bands). The degeneration which occurs as $a_{z}$ decreases can also be deduced from the illustrated electric field maps in Fig. 8(a), 8(c), and 8(e). The stacking effect between the resonators is the most efficient when $a_{z}=2 \mathrm{~mm}$. The intensified fields are distributed symmetrically for each layer. The fields are less interacting in Fig. 8(a) for $a_{z}=$ $6 \mathrm{~mm}$ and the intensified field amplitudes drop along the propagation direction due to the accompanying losses with each stacked layer. Then again, the multimode operation produces an asymmetrical field map for $a_{z}=1 \mathrm{~mm}$ case in Fig. 8(e) owing to the interference of the modes with negative and positive phase advance characteristics. In the end, the re-homogenized configuration $\left(a_{z}=1 \mathrm{~mm}\right.$ case) does not accurately yield a well-established NPV transmission band.

\subsection{Simultaneously Opening NPV and PPV Transmission Channels with the Dense Configuration}

Both of these bands can be functionalized at the same time when the hybrid unit lattice is adapted and a defect is formed by removing one layer in the middle. The transmission results for this case are portrayed in Fig. 10. The experimental validation of the relevant transmission channels are also plotted on the same graph (blue line). It is verified that there is a good connection between the experimental and numerical results. The slight shift in the frequency domain is attributed to both the differences in the material properties that are provided by the supplier in contrast to the modeled structure in the simulations (e.g., the permittivity of Teflon substrate, spatial shifts between the metallic patterns on both faces) and difficulties in the manual alignment of the stacked layers.

The mentioned spatial shift of the metallic patterns on both faces of the same layer also causes unavoidable misalignment in the experiments. The effect of the oblique incidence

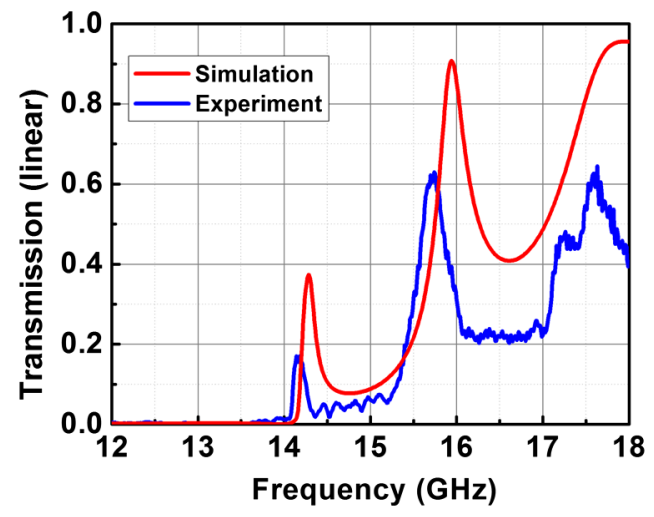

Fig. 10 The demonstration of the simultaneous opening of the NPV and PPV transmission channels on the transmission spectrum. Numerical results are plotted in red color and measurement results are depicted in blue color. 
that mimics our misalignment problem in our experiments had severely caused nonidealities in Ref. 34. The oblique incidence had triggered shifts in the frequency spectrum owing to the anisotropic nature of the stacked fishnet layers. Previous studies with hybrid unit lattice configurations had also shown that even a small tilting of the incident wave could destroy the NPV transmission band while pulling down the transmission values. ${ }^{23}$

Regardless of these secondary factors, two transmission channels with negative and positive phase propagation characteristics have been observed for the same sample configuration at previously impenetrable frequency values.

\subsection{Scaled Design at the Optical Regime}

The considered structures can be designed at optical wavelengths owing to the scalability of Maxwell's equations. Likewise, Fig. 11 studies the arrangements at the optical regime. Two main configurations that have been investigated throughout the present paper are modeled in Fig. 11(a) and 11(b). The metal thickness, $t_{m}=40 \mathrm{~nm}$ for both structures. The subwavelength

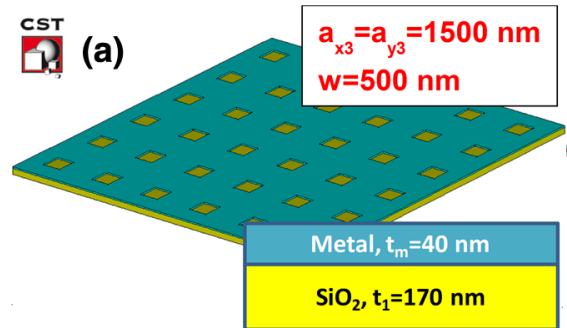

(c)

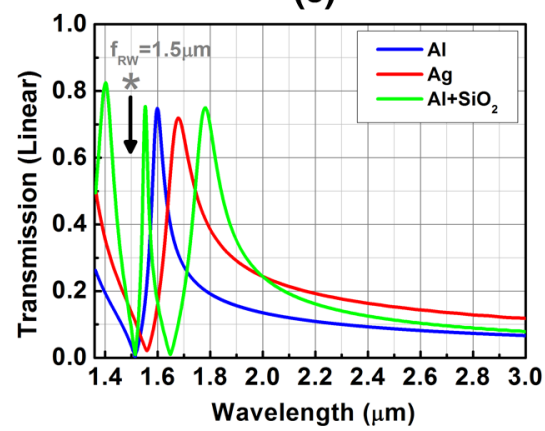

(e)

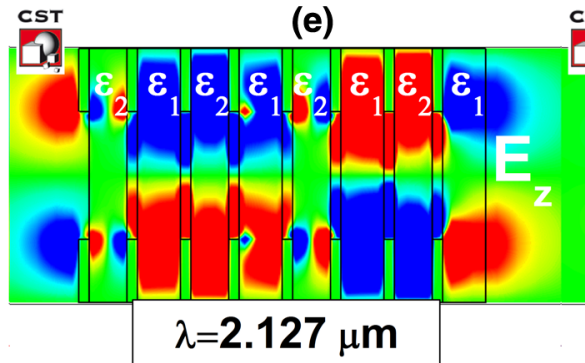

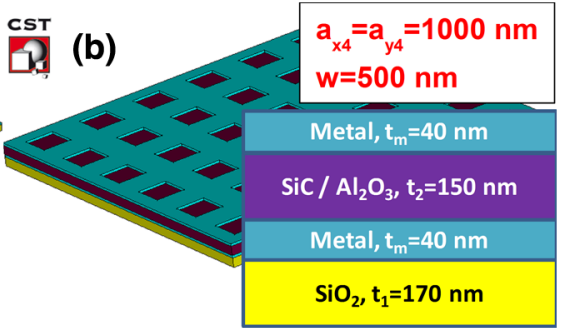

(d)

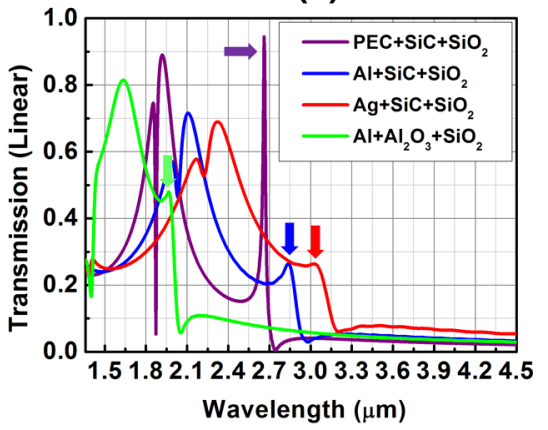

(f)

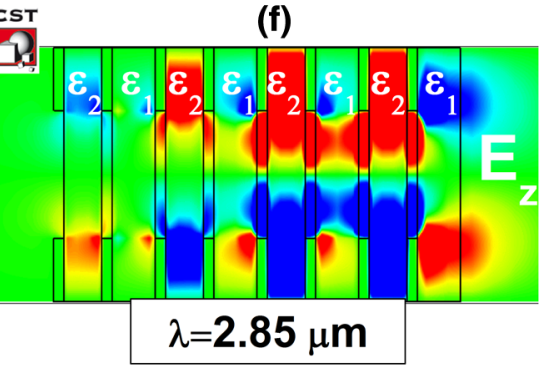

Fig. 11 The considered configurations at optical wavelengths. (a) The first configuration and (b) the hybrid configuration. The regarding deposited layers are shown in the figure insets with their relevant thicknesses. (c) The transmission spectrum for the first configuration with Al (blue line), Ag (red line). Aluminum pattern is developed on top of the glass substrate (green line). (d) The transmission spectrum for the second configuration with different metallic and sandwiched dielectric layers, $\mathrm{PEC}+\mathrm{SiC}$ (purple line), $\mathrm{Al}+\mathrm{SiC}$ (blue line), $\mathrm{Ag}+\mathrm{SiC}$ (red line), $\mathrm{Al}+\mathrm{Al}_{2} \mathrm{O}_{3}$ (green line). The color-coded arrows indicate the peak points of the NPV transmission bands, which are located at $\lambda_{\mathrm{PEC}}=2.658 \mu \mathrm{m}, \quad \lambda_{\mathrm{Al}+\mathrm{SiC}}=2.845 \mu \mathrm{m}, \quad \lambda_{\mathrm{Ag}+\mathrm{SiC}}=3.037 \mu \mathrm{m}$, $\lambda_{\mathrm{Al}+\mathrm{Al}_{2} \mathrm{O}_{3}}=1.958 \mu \mathrm{m}$. The steady state field distributions $\left(E_{z}\right)$ for the five-layer hybrid structure $(\mathrm{Al}+\mathrm{SiC})$ at (e) $2.127 \mu \mathrm{m}$ and (f) $2.85 \mu \mathrm{m}$ (Video 5). The incoming field is launched from the right hand side. (Video 5, MPEG, 828 KB). [URL: http://dx.doi.org/10.1117/1.JNP.6.1.061608.5]. 
aperture arrays have a square opening whose side $w=500 \mathrm{~nm}$, again for both cases in Fig. 11(a) and 11(b). Such apertures can be patterned with the aid of the electron beam or focused ion beam lithography techniques on the metal layers. However, the metallic patterns can no longer remain suspended in air. The volume that is occupied by the air spacing in the microwave configurations is replaced with the glass substrate $\left(\varepsilon_{1}=2.25\right)$ with a thickness, $t_{1}=170 \mathrm{~nm}$.

The configuration in Fig. 11(a) is the homogenous unit lattice design whose transmission was governed by the higher order diffractions. The lattice constants on the transversal plane, $a_{x 3}=a_{y 3}=1500 \mathrm{~nm}$. The regarding transmission spectrum is given in Fig. 11(c). First, the simulations are carried out with aluminum (blue line) and silver (red line) metallic layers. Then, the effect of the glass substrate is studied. Drude models have been adapted for the metals, where $\omega_{\mathrm{pAl}}=2.4 \times 10^{16} \mathrm{rad} / \mathrm{sn}, \omega_{\mathrm{pAg}}=1.325 \times 10^{16} \mathrm{rad} / \mathrm{sn}, \quad v_{\mathrm{cAl}}=$ $175 \mathrm{THz}, v_{\mathrm{cAg}}=130 \mathrm{THz}$ and $\varepsilon_{\infty \mathrm{Al}}=\varepsilon_{\infty \mathrm{Ag}}=1$. The classical features of the transmission spectrum dominated by diffractions are evident in Fig. 11(c). The corresponding RW anomaly should be located at $\lambda_{\mathrm{RW}}=a_{x 3}=a_{y 3}=1500 \mathrm{~nm}$ for the case where perfect metal layers are involved. The effect of the plasmons causes a slight red shift in the RW anomaly. Since Al has a higher plasma frequency, this red shift is less pronounced in comparison to the case with $\mathrm{Ag}$ (compare blue and red colored lines around $1.5 \mu \mathrm{m}$ ). In accordance with the earlier claims and designs in the microwave domain, the EOT emerges just after the RW anomaly (i.e., at a longer wavelength) and transmission values exceeding $70 \%$ can be detected. Again as in the case of the studied microwave examples, the glass substrate underneath the metallic pattern caused another red shift in the EOT (green line). This shift in the EOT wavelength is calculated to be as high as $0.18 \mu \mathrm{m}$ (compare the peak transmission levels of blue and green colored lines).

Conversely, the transmission spectrum is given for the hybrid unit lattice configuration in Fig. 11(d). A secondary dielectric medium is sandwiched between two metallic patterns, as in the case of the microwave designs. The thickness of the second dielectric medium is chosen to be $t_{2}=150 \mathrm{~nm}$. The sandwiched dielectric layer must have a higher dielectric permittivity than the glass substrate. Accordingly, $\mathrm{SiC}$ and $\mathrm{Al}_{2} \mathrm{O}_{3}$ with $\varepsilon_{\mathrm{SiC}}=6.708, \varepsilon_{\mathrm{Al}_{2} \mathrm{O}_{3}}=3.132$ at nearinfrared wavelengths have been utilized. The stacked layers are shown in Fig. 11(b). The first metallic pattern ( $\mathrm{Al}$ or $\mathrm{Ag})$ is deposited on top of the glass substrate, whereas the second metallic pattern at the topmost is developed on top of the secondary dielectric medium $\left(\mathrm{SiC}\right.$ or $\left.\mathrm{Al}_{2} \mathrm{O}_{3}\right)$ with a relatively high index.

The NPV transmission band's peaks appear at wavelengths designated by the color-coded arrows in Fig. 11(d). Then again, the NPV transmission band could also be achieved theoretically with the employment of a perfect electric conductor (PEC) at these wavelengths, too [see purple colored line in Fig. 11(d)]. We do not have a PEC at optical wavelengths and the metals have dispersive characteristics, but the presence of the NPV transmission band with a purely theoretical PEC material at near-infrared frequencies shows that the observed peak is not invoked by the special features of the surface plasmon polaritons (SPP). Instead, the observed NPV transmission band with the PEC material is the manifestation of the same physics that are investigated in details at microwave frequencies. Correspondingly, we can use the proportionality offered by Eq. (3) in order to determine the specific location of the peak of the NPV transmission band on the wavelength axis,

$$
\lambda_{\mathrm{PEC}}=\frac{c}{f_{\mathrm{GHz}}} \times \frac{\sqrt{\frac{1}{\varepsilon_{\text {teflon }}} \times\left[\frac{1}{a_{y 2}\left(a_{y 2}-w\right)}+\frac{\left(a_{x 2}-w_{\mathrm{GHz}}\right)}{a_{x 2} a_{y 2} w_{\mathrm{GHz}}}\right]}}{\sqrt{\frac{1}{\varepsilon_{\mathrm{SiC}}} \times\left[\frac{1}{a_{y 4}\left(a_{y 4}-w\right)}+\frac{\left(a_{x 4}-w\right)}{a_{x 4} a_{y 4} w}\right]}},
$$

where $c$ is the speed of light and the relevant dimensions of the hybrid configuration (second configuration) are used. Once we carry out the algebra and insert the values $f_{\mathrm{GHz}}=14.27 \mathrm{GHz}$, $\varepsilon_{\text {teflon }}=2.16, a_{x 2}=a_{y 2}=14 \mathrm{~mm}$ and $w_{\mathrm{GHz}}=7 \mathrm{~mm}$, the envisaged NPV transmission band's peak turns out to be at $\lambda_{\mathrm{PEC}}=2.646 \mu \mathrm{m}$ when $\mathrm{SiC}$ layer is sandwiched between the metallic patterns. On the other hand, the NPV transmission band's peak is located at $2.658 \mu \mathrm{m}$ in Fig. 11(d) with the PEC (see the purple arrow), which is a very close value to the estimated one by using the simple $L C$ resonator. 
Then again, the real metals have to be taken into account at optical wavelengths. The excited SPP once again causes a red shift with respect to the PEC case. The detected NPV transmission band's peaks are located at $2.845 \mu \mathrm{m}$ and $3.037 \mu \mathrm{m}$ for Al and Ag, respectively. The NPV bands are followed by the PPV bands in Fig. 11(d) regardless of the material selections. The PEC case yields the highest transmission figures within the NPV transmission band. Particularly, the transmission peak value is increased more than three times with the PEC configuration in contrast to the real metal applications. From this point of view, the plasmonic effects have a degrading effect on the performance characteristics. In summary, the entailed plasmonic effects in the resonator structure cause a red shift in the NPV transmission band's peak, decrease the detected peak values of the NPV bands, broaden the NPV bands and tend to destroy the valley between the NPV and PPV bands by tending to merge the initially separated NPV and PPV bands.

Aluminum turns out to be a better candidate than the silver option as a metal deposition layer if we compare the NPV transmission bands in Fig. 11(d). The NPV band is more isolated from the PPV band when $\mathrm{Al}$ is selected due to the higher plasma frequency of Al. Then, a multimode operation with the concurrent negative and positive phase advance, which was observed at the microwave frequencies with the collapse of the PPV band into the NPV band [see $a_{z}=1 \mathrm{~mm}$ case in Fig. 9(b)] is less likely to happen even with the employment of different dielectric media when $\mathrm{Al}$ is employed. Nevertheless, the difficulties of the microfabrication related to $\mathrm{Al}$ manufacturing have to be resolved with alternative methods.

Following the formalism in Ref. 27, the location of the NPV transmission bands can be roughly estimated. The conservation of the momentum at the metallic interface dictates that

$$
k_{\mathrm{SPP}}=k_{0} \sin (\phi)+\frac{2 \pi}{a_{x 4}} m+\frac{2 \pi}{a_{y 4}} n,
$$

where $k_{\mathrm{SPP}}$ is the wave vector of the SPP (in scalar format), $k_{0}$ is the wave vector of the incident light, $\phi$ is the incident angle, $m$ and $n$ are the integer values. For the incoming light at normal incident $(\phi=0)$, the mode with the largest wavelength ( $m=1$ and $n=0$ or $m=0$ and $n=1)$ is located at $\lambda_{\mathrm{SPP}}=a_{x 4}=a_{y 4}$. This value is equal to the formerly introduced $f_{\mathrm{RW}}$ for this design.

On the other hand, at large wavelengths (as in our case) the following formula can be used to find the relation between $\lambda_{\mathrm{SPP}}$ and $\lambda_{0}$

$$
\lambda_{0}=\lambda_{\mathrm{SPP}} \sqrt{\varepsilon_{2} \times \frac{t_{2}+2 \operatorname{coth}\left(\omega_{p} t_{m} / c\right) /\left(\omega_{p} / c\right)}{t_{2}}} .
$$

The sandwiched dielectric medium is $\mathrm{SiC}$, and the peak values of transmission are calculated to occur at $2.798 \mu \mathrm{m}$ and $2.976 \mu \mathrm{m}$ for $\mathrm{Al}$ and $\mathrm{Ag}$, respectively. These are good estimates of the simulated NPV transmission band's peak locations. The peak is calculated at $1.912 \mu \mathrm{m}$ by using Eq. (6) when $\mathrm{Al}_{2} \mathrm{O}_{3}$ is used as the secondary dielectric layer instead of SiC. The corresponding peak value is found at $1.958 \mu \mathrm{m}$ (green arrow) in the simulated transmission spectrum in Fig. 11(d). The peak values of the simulation results with $\mathrm{SiC}(2.845 \mu \mathrm{m})$ and $\mathrm{Al}_{2} \mathrm{O}_{3}$ $(1.958 \mu \mathrm{m})$ secondary dielectric layers do scale with $\sqrt{\varepsilon_{\mathrm{SiC}} / \varepsilon_{\mathrm{Al}_{2} \mathrm{O}_{3}}}$ in accordance with both Eqs. (3) and (6) for the designs employing Al metallic layers. The NPV band is not as separated from the PPV band as in the case of the $\mathrm{SiC}$ choice for the $\mathrm{Al}_{2} \mathrm{O}_{3}$ case in Fig. 11(d). Yet, the NPV band provides higher transmission at smaller wavelengths for the $\mathrm{Al}_{2} \mathrm{O}_{3}$ case.

Finally, the electric field maps $\left(E_{z}\right)$ for the five-layer hybrid configuration at specific wavelengths that are chosen from the PPV and NPV bands are depicted in Fig. 11(e) and 11(f), respectively. The negative phase advance can be monitored in the linked movie file (Video 5) while the incident light is approaching to the periodic arrangement from the righthand side. It can be immediately noticed that the fields are localized in the higher permittivity layers due to the resonator nature of the stacked layers in Fig. 11(f), whereas the fields do not have a specific preference at the PPV band in Fig. 11(e). This is a very similar situation that we had encountered in the previous sections for the NPV transmission band at microwave frequencies [e.g., Figs. 6(d) and 7(d)]. 


\section{Conclusion}

As a summary, we have started our analyses with two similar configurations with subwavelength apertures. One of the configurations had a relatively dense periodic arrangement in comparison to the other. We have first simulated the transmission spectrum for these designs with homogenous unit lattices. Several design variations of the first configuration were already inspected in the literature under the framework of the EOT through subwavelength apertures. The physical origins of the emerging EOT band have been discussed by borrowing the established theoretical background from the literature, and it is shown that the EOT band is achieved with the aid of the higher diffraction orders of the artificial waveguide surrounding the unit lattice. Conversely, the dense configuration did not yield a NPV transmission band in its original form.

First, a PPV transmission band that is based on the FP cavity formation is examined for the dense configuration. Later on, a NPV transmission band is also realized by hybridizing the spatial distribution of the unit lattice along the propagation direction. The underlying physics of this type of wave propagation is studied with the help of the field maps and dispersion diagrams. It has been shown that the electromagnetic wave propagation inside the NPV transmission band for the dense configuration is governed by the stacked resonators that are coupled to each other. Thus, the origins of the NPV band are different in each case (for the first and second configurations). We conclude that the EOT does not heavily rely on the spatial arrangement of the unit lattice (for a fixed value of the unit lattice separation along the propagation direction that would allow negative phase advance) as long as the contribution of the higher diffraction orders is guaranteed for the first configuration, whereas the fractions of the volume occupied by different dielectric media play a key factor for the hybridized unit lattice orientations of the dense configuration.

As a final point, the issue of the scalability of these configurations to the optical wavelengths has been addressed. The NPV transmission band has been demonstrated in the near-infrared region. In general, the plasmonic effects have been shown to degrade the performance of the stacked resonators. Nevertheless, the NPV band based on the resonator nature of the hybrid unit lattice is still achievable at optical wavelengths once the fabrication difficulties are overcome. Different dielectric and metallic layers have been simulated, and their influence on the performance characteristics has been discussed.

The hybrid unit lattices can be exploited as the building blocks to construct a brand new prism or a demultiplexer that could potentially be used at optical frequencies. The microwave experiments show that the losses are mainly due to the included dielectric loading. Yet, the discussed coupling and stacking effects might be advantageous or disadvantageous depending on the application field. A designer has to carefully take into consideration the aforementioned effects before building a device with the hybrid configurations. Some of the promising devices that offer and introduce interesting new features have already been scaled up to optical wavelengths by the metamaterial research community once the researchers fully explained the underlying physical mechanisms and shed light on the possible performance-related deteriorating factors, as we have attempted to do throughout the present paper.

\section{Acknowledgments}

This work is supported by the projects DPT-HAMIT, ESF-EPIGRAT, EU-N4E, NATO-SET-181 and TUBITAK under Project Nos., 107A004, 107A012, 109E301. One of the authors (E.O.) also acknowledges partial support from the Turkish Academy of Sciences.

\section{References}

1. H. A. Bethe, "Theory of diffraction by small holes," Phys. Rev. 66(7-8), 163-182 (1944), http://dx.doi.org/10.1103/PhysRev.66.163.

2. T. W. Ebbesen et al., "Extraordinary optical transmission through sub-wavelength hole arrays," Nature 391(6668), 667-669 (1998), http://dx.doi.org/10.1038/35570. 
3. L. Martin-Moreno et al., "Theory of extraordinary optical transmission through subwavelength hole arrays," Phys. Rev. Lett. 86(6), 1114-1117 (2001), http://dx.doi.org/10.1103/ PhysRevLett.86.1114.

4. J. A. Porto, F. J. Garcia-Vidal, and J. B. Pendry, "Transmission resonances on metallic gratings with very narrow slits," Phys. Rev. Lett. 83(14), 2845-2848 (1999), http://dx.doi.org/10 .1103/PhysRevLett.83.2845.

5. K. G. Lee and Q. -H. Park, "Coupling of surface plasmon polaritons and light in metallic nanoslits," Phys. Rev. Lett. 95(10), 103902 (2005), http://dx.doi.org/10.1103/PhysRevLett .95 .103902 .

6. M. Beruete et al., "Enhanced millimeter wave transmission through quasioptical subwavelength perforated plates," IEEE Trans. on Anten. and Propag. 53(6), 1897-1903 (2005), http://dx.doi.org/10.1109/TAP.2005.848689.

7. F. J. Garcia-Vidal et al., "Multiple paths to enhance optical transmission through a single subwavelength slit," Phys. Rev. Lett. 90(21), 213901 (2003), http://dx.doi.org/10.1103/ PhysRevLett.90.213901.

8. J. B. Pendry, L. Martin-Moreno, and F. J. Garcia-Vidal, "Mimicking surface plasmons with structured surfaces," Science 305(5685), 847-848 (2004), http://dx.doi.org/10.1126/science .1098999.

9. R. Marques et al., "Analytical theory of extraordinary transmission through metallic diffraction screens perforated by small holes," Opt. Express 17(7), 5571-5579 (2009), http://dx .doi.org/10.1364/OE.17.005571.

10. F. Medina, F. Mesa, and R. Marques, "Extraordinary transmission through arrays of electrically small holes from a circuit theory perspective," IEEE Trans. Microw. Theory Tech. 56(12), 3108-3120 (2008), http://dx.doi.org/10.1109/TMTT.2008.2007343.

11. M. Beruete et al., "Molding left- or right-handed metamaterials by stacked cutoff metallic hole arrays," IEEE Trans. on Anten. and Propag. 55(6), 1514-1521 (2007), http://dx.doi .org/10.1109/TAP.2007.897324.

12. A. Karabchevsky, M. Auslender, and I. Abdulhalim, "Dual-surface plasmon excitation with thin metallic nanoslits," J. Nanophoton. 5(1), 051821 (2011), http://dx.doi.org/10.1117/1.3609276.

13. G. Dolling et al., "Negative-index metamaterial at $780 \mathrm{~nm}$ wavelength," Opt. Lett. 32(1), 53-55 (2007), http://dx.doi.org/10.1364/OL.32.000053.

14. G. Dolling et al., "Simultaneous negative phase and group velocity of light in a metamaterial," Science 312(5775), 892-894 (2006), http://dx.doi.org/10.1126/science.1126021.

15. M. Beruete, M. Sorolla, and I. Campillo, "Left-handed extraordinary optical transmission through a photonic crystal of subwavelength hole arrays," Opt. Express 14(12), 5445-5455 (2006), http://dx.doi.org/10.1364/OE.14.005445.

16. M. Beruete et al., "Connection between extraordinary transmission and negative refraction in a prism of stacked sub-wavelength hole arrays," J. Phys. D: Appl. Phys. 42(16), 165504 (2009), http://dx.doi.org/10.1088/0022-3727/42/16/165504.

17. C. Menzel et al., "Validity of effective material parameters for optical fishnet metamaterials," Phys. Rev. B 81(3), 035320 (2010), http://dx.doi.org/10.1103/PhysRevB.81.035320.

18. L. Jelinek, R. Marques, and J. Machac, "Fishnet metamaterials-rules for refraction and limits of homogenization," Opt. Express 18(17), 17940-17948 (2010), http://dx.doi.org/10 .1364/OE.18.017940.

19. G. V. Eleftheriades, O. Siddiqui, and A. K. Iyer, "Transmission line models for negative refractive index media and associated implementations without excess resonators," IEEE Microw. Wirel. Compon. Lett. 13(2), 51-53 (2003), http://dx.doi.org/10.1109/LMWC .2003.808719.

20. M. Beruete et al., "Increase of the transmission in cut-off metallic hole arrays," IEEE Microw. Wirel. Compon. Lett. 15(2), 116-118 (2005), http://dx.doi.org/10.1109/LMWC .2004 .842852 .

21. M. Beruete et al., "Enhanced millimeter-wave transmission through subwavelength hole arrays," Opt. Lett. 29(21), 2500-2502 (2004), http://dx.doi.org/10.1364/OL.29.002500.

22. K. B. Alici and E. Ozbay, "A planar metamaterial: polarization independent fishnet structure," Photon. Nano. Fund. Appl. 6(1), 102-107 (2008), http://dx.doi.org/10.1016/j .photonics.2008.01.001. 
23. K. B. Alici and E. Ozbay, "Characterization and tilted response of a fishnet metamaterial operating at $100 \mathrm{GHz}$, ' J. Phys. D: Appl. Phys. 41(13), 135011 (2008), http://dx.doi.org/10 .1088/0022-3727/41/13/135011.

24. N. -H. Shen et al., "Parametric investigation and analysis of fishnet metamaterials in the microwave regime," J. Opt. Soc. Am. B 26(12), B61-B67 (2009), http://dx.doi.org/10 .1364/JOSAB.26.000B61.

25. K. Aydin et al., "Negative phase advance in polarization independent, multi-layer negativeindex metamaterials," Opt. Express 16(12), 8835-8843 (2008), http://dx.doi.org/10.1364/ OE.16.008835.

26. M. Kafesaki et al., "Left-handed metamaterials: the fishnet structure and its variations," Phys. Rev. B 75(23), 235114 (2007), http://dx.doi.org/10.1103/PhysRevB.75 .235114.

27. R. Ortuno et al., "Role of surface plasmon polariton on extraordinary optical transmission through double-layer metallic hole arrays," Phys. Rev. B 79(7), 075425 (2009), http://dx.doi .org/10.1103/PhysRevB.79.075425.

28. R. Marques et al., "Analytical theory of wave propagation through stacked fishnet metamaterials," Opt. Express 17(14), 11582-11592 (2009), http://dx.doi.org/10.1364/OE.17 .011582 .

29. H. Caglayan et al., "Experimental observation of cavity formation in composite metamaterials," Opt. Express 16(15), 11132-11140 (2008), http://dx.doi.org/10.1364/OE.16 .011132 .

30. M. Beruete et al., "Circuit approach to the minimal configuration of terahertz anomalous extraordinary transmission,” Appl. Phys. Lett. 98(1), 014106 (2011), http://dx.doi.org/10 $.1063 / 1.3533815$.

31. M. Beruete, M. Navarro-Cia, and M. Sorolla, "Understanding anomalous extraordinary transmission from equivalent circuit and grounded slab concepts," IEEE Trans. Microw. Theory Tech. 59(9), 2180-2188 (2011), http://dx.doi.org/10.1109/TMTT.2011 .2160076 .

32. M. Beruete et al., "Extraordinary transmission and left-handed propagation in miniaturized stacks of doubly periodic subwavelength hole arrays," Opt. Express 15(3), 1107-1114 (2007), http://dx.doi.org/10.1364/OE.15.001107.

33. J. Zhou et al., "Size dependence and convergence of the retrieval parameters of metamaterials," Photon. Nano. Fund. Appl. 6(1), 96-101 (2008), http://dx.doi.org/10.1016/j .photonics.2007.10.003.

34. M. Beruete, M. Navarro-Cia, and M. Sorolla, "Strong lateral displacement in polarization anisotropic extraordinary transmission metamaterial," New J. Phys. 12(6), 063037 (2010), http://dx.doi.org/10.1088/1367-2630/12/6/063037.

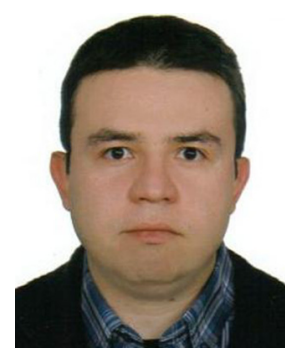

Atilla Ozgur Cakmak received his BSc in microelectronics engineering with a minor in physics in 2003 from Sabanci University (Istanbul, Turkey). He received his MSc degree in computer science and electronics engineering again from Sabanci University in 2005. He has recently received his PhD from Bilkent University (Ankara, Turkey) from the electrical and electronics engineering department in 2012. His research interests include photonic crystals, nanometamaterials, nanophotonics, nanoplasmonics, and analytical models in electromagnetic for the periodic structures.

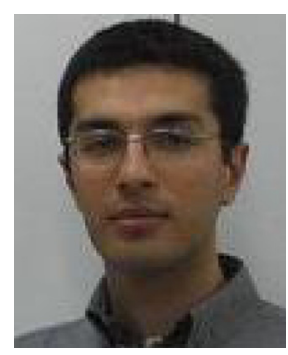

Evrim Colak received his BSc in electrical and electronics engineering (EEE) and in physics in 2002 from Middle East Technical University (METU, Ankara Turkey). He received his MSc degree in biomedical imaging from the EEE department at METU in 2005. He has recently received his PhD in Bilkent University (Ankara, Turkey), pursuing research on nanometamaterials, nanophotonics, and photonic crystals in 2012. 


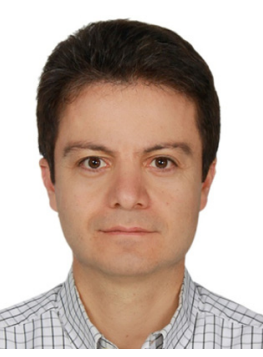

Ekmel Ozbay received MS and PhD degrees from Stanford University in electrical engineering, in 1989 and 1992. He joined Bilkent University (Ankara, Turkey) in 1995, where he is currently a full professor in the Department of Electrical-Electronics Engineering. His research in Bilkent involves nanophotonics, nanometamaterials, nanoplasmonics, nanodevices, photonic crystals, and GaN/AlGaN MOCVD growth. He is the 1997 recipient of the Adolph Lomb Medal of the Optical Society of America and the 2005 European Union Descartes Science award. He worked as an editor for Optics Letters, Photonics and Nanostructures and IEEE Journal of Quantum Electronics journals. He has published 295 articles in SCI journals. His papers have received $7400+$ citations with an $h$-index of 42 . 